\title{
NOTE ON STRUCTURAL STABILITY
}

\author{
BY J. L. ARRAUT ${ }^{1}$
}

Communicated by H. A. Antosiewicz, January 13, 1966

Introduction. In this note we prove that if $X$ is a structural stable vector field on a compact manifold $M$ of dimension $m \geqq 2$, then any real valued function defined on $M$ which is invariant under $X$ i.e., which is constant along orbits of $X$, must be constant. Of course some assumptions of differentiability are made. From this follows in particular that structural stable vector fields do not admit global first integrals. Here we use the $\epsilon$-definition of structural stability, for the non $\epsilon$-definition (see [1]) it is still a problem.

1. We will denote by $M$ a connected compact manifold of dimension $m \geqq 2$, with a differentiable structure of class large enough for our purpose. Let $\mathfrak{F}_{k}(M)$ be the set of all real valued functions of class $C^{k}, k \geqq 1$, defined on $M$ and $\mathfrak{x}_{k}(M)$ the space of all $C^{k}$ vector fields on $M$ with the $C^{k}$-topology. Finally let $\Sigma_{k}(M)$ be the set of structural stable vector fields contained in $\mathfrak{x}_{k}(M)$. (See [1].)

Let $X \in \mathfrak{x}_{k}(M)$. A function $f \in \mathfrak{F}_{k}(M)$ is called invariant under $X$ if it is constant along orbits of $X$. It is equivalent to say that $X f=0$, where $X f$ denotes differentiation of $f$ with respect to the vector field $X$. An invariant function $f$ under $X$ is called a global first integral of $X$ if it is nonconstant on any open subset of $M$.

Lemma 1. If $f \in F_{k}(M) k \geqq 1$, then the set $\Gamma$ of critical values of $f$ is closed in $R$.

Let $X \in \mathfrak{x}_{k}(M) k \geqq 1$ and $f \in \mathfrak{F}_{k+1}(M)$ a nonconstant function invariant under $X$. We assume that $f$ has at least one regular value and from Lemma 1 it follows then, that there is an open set of them. The inverse image $f^{-1}(a)$ of every regular value $a$ of $f$ is a compact manifold $N_{a}$ of dimension $(m-1)$ properly imbedded in $M$. Since $f$ is invariant under $X$ every $N_{a}$ is invariant under $X$, i.e., $N_{a}$ is a union of orbits of $X$. Let $\left[a_{1}, a_{2}\right]$ be a closed interval made up of regular values of $f$, we are going to define a special kind of perturbation of $X$ associated to the interval $\left[a_{1}, a_{2}\right]$.

Fix a positive definite Riemannian metric $g$ on $M$ of differentiability class large enough. Let $\operatorname{grad} f$ be the vector field on $M$ defined by the equation

${ }^{1}$ This research was supported by the United States Army Research Office, Durham under Contract No. DA-31-124-ARD-D-270. 


$$
Y f=g(Y, \operatorname{grad} f)
$$

where $Y$ is any vector field on $M$. Let $h$ be a real valued function of class $C^{\infty}$ defined on $R$ such that $h>0$ on $\left(a_{1}, a_{2}\right)$ and $h=0$ otherwise. Then $h \circ f \in \mathcal{F}_{k+1}(M), h \circ f>0$ on the open submanifold $P=f^{-1}\left(\left(a_{1}, a_{2}\right)\right)$ and $h \circ f=0$ otherwise. Finally for every real number $\alpha>0$ we define the vector field

$$
X_{\alpha}=X+\alpha(h \circ f)(\operatorname{grad} f) .
$$

Lemma 2. $X_{\alpha}$ for $\alpha>0$ has the following properties:

1. $X_{\alpha} \in \mathfrak{x}_{k}(M)$;

2. $X_{\alpha}=X$ outside $P$. In particular $N_{a_{1}}$ and $N_{a_{2}}$ are invariant under $X_{\alpha}$;

3. $\left(X_{\alpha}\right)_{p} \neq 0$ at every point $p \in P$ or in other words $X_{\alpha}$ has no singularities on $P$.

4. $\left(X_{\alpha}\right)_{p} f>0$ at every point $p \in P$.

Properties 1, 2, and 3 follow straight from the definition of $X_{\alpha}$. A direct computation proves Property 4. In fact for any $p \in P$ we have

$$
\left(X_{\alpha}\right)_{p} f=(X+\alpha(h \circ f)(\operatorname{grad} f))_{p} f=X_{p} f+\alpha(h \circ f)(p)(\operatorname{grad} f)_{p} f .
$$

But $\alpha>0,(h \circ f)(p)>0$ and $(\operatorname{grad} f)_{x} f=g_{p}(\operatorname{grad} f, \operatorname{grad} f)>0$, thus $\left(X_{\alpha}\right)_{p} f>0$.

Lemma 3. For every point $p \in P$ the $\omega$-limit set ( $\alpha$-limit set) of $p$ with respect to $X_{\alpha}$ is contained in $N_{a_{2}}\left(N_{a_{1}}\right)$.

Let $\psi_{t}$ denote the 1-parameter group of transformations associated with $X_{\alpha}$. For every fixed point $p \in P$ we consider the function $f\left(\psi_{t}(p)\right)$ as a function of $t$ for $t \geqq 0$. From Property 4 of Lemma 2 it follows that $f\left(\psi_{t}(p)\right)$ is a strictly increasing function of $t$. On the other hand $f\left(\psi_{t}(p)\right)$ can never reach the value $a_{2}$, because if for some $t_{0}>0, f\left(\psi_{t}(p)\right)=a_{2}$, then $\psi_{t_{0}}(p) \in N_{a_{2}}$ and since $N_{a_{3}}$ is invariant under $X_{a}$, it would imply $p \in N_{a_{2}}$. We are going to show that $f\left(\psi_{t}(p)\right)$ tends to $a_{2}$ as $t$ tends to $+\infty$. Suppose $\lim f\left(\psi_{t}(p)\right)=a<a_{2}$, then by the continuity of $f$ it follows that $\omega(p) \subset N_{a}$. But this contradicts the fact that $f\left(\psi_{t}(q)\right)$ is strictly increasing for every $q \in P$. Thus $\lim f\left(\psi_{t}(p)\right)$ must be $a_{2}$. As it was observed above, the continuity of $f$ implies $\omega(p) \subset N_{a_{2}}$. The proof for the $\alpha$-limit set is the same.

PROPOSITION. If $X \in \Sigma_{k}(M) k \geqq 1$, then there is no function $f \in F_{k+1}(M)$ such that:

(i) $f$ is invariant under $X$.

(ii) $f$ is nonconstant and has at least a regular value. 
Suppose there is a function $f \in \mathfrak{F}_{k+1}(M)$ satisfying (i) and (ii). Since $f$ has a regular value, it has a closed interval of them $\left[a_{1}, a_{2}\right]$. Consider the family $X_{\alpha}$ of vector fields associated with the interval $\left[a_{1}, a_{2}\right]$. Let $a=\frac{1}{2}\left(a_{1}+a_{2}\right)$ and $\eta$ the distance between $N_{a}$ and $N_{a_{1}} \cup N_{a_{2}}$. Assume now that $X$ is structural stable, i.e. given $\epsilon>0$ there exists $\delta>0$ such that $Y \in \mathfrak{x}_{k}(M)$ and $d(Y, X)<\delta$ implies there is a $\epsilon$-homeomorphism $\phi$ of $M$ onto $M$ which sends orbits of $X$ into orbits of $Y$. Take $\epsilon<\eta / 2$ and $\alpha$ such that $d\left(X_{\alpha}, X\right)<\delta$, the corresponding homeomorphism $\phi$ will move every point of $N_{a}$ less than $\epsilon$ and so the distance between $\phi\left(N_{a}\right)$ and $N_{a_{1}} \cup N_{a_{2}}$ will be greater than $\eta / 2$. Moreover $\phi\left(N_{a}\right) \subset P$ and is invariant under $X_{\alpha}$, but this is a contradiction with Lemma 3.

Corollary. If $X \in \Sigma_{k}(M) k \geqq 1$, then $X$ does not admit a global first integral $f \in \mathcal{F}_{k+1}(M)$ with a regular value.

REMARK. If we use Sard's theorem (see [2]) to establish the existence of regular values for a differentiable function $f$ of $M$ into $R$, then the proposition and the corollary can be set in the following way:

Proposition. If $X \in \Sigma_{m}(M)$ where $m=\operatorname{dim} M$, then any function $f \in \mathfrak{F}_{m+1}(M)$ which is invariant under $X$ must be constant.

Corollary. If $X \in \Sigma_{m}(M)$ where $m=\operatorname{dim} M$, then $X$ does not admit a global first integral $f \in \mathcal{F}_{m+1}(M)$.

\section{BIBLIOGRAPHY}

1. M. Peixoto, Structural stability on two-dimensional manifolds, Topology 1 (1962), 101-120.

2. A. Sard, The measure of the critical values of differentiable maps, Bull. Amer. Math. Soc. 48 (1942), 883-890.

BROWN UNIVERSITY AND

Centro de Investigación del. I.P.N., Mexico 